\title{
On Binomial Set-Theoretic Complete Intersections in Characteristic $p$
}

\author{
Margherita BARILE \\ Dipartimento di Matematica \\ Università di Bari \\ Via E. Orabona 4 \\ 70125 Bari - Italy \\ barile@dm.uniba.it
}

Received: September 9, 2007

Accepted: October 15, 2007

\begin{abstract}
Using arithmetic conditions on affine semigroups we prove that for a simplicial toric variety of codimension 2 the property of being a set-theoretic complete intersection on binomials in characteristic $p$ holds either for all primes $p$, or for no prime $p$, or for exactly one prime $p$.
\end{abstract}

Key words: toric variety, set-theoretic complete intersection, affine semigroup, $p$-gluing. 2000 Mathematics Subject Classification: 14M10; 13E15, 14M25, $20 \mathrm{M} 05$.

\section{Introduction}

It has been recently discovered that the minimum number of equations that are needed to define an algebraic variety, in general, depends on the characteristic of the ground field. The first examples to be found were the determinantal and Pfaffian varieties considered in [1]. Later, in [2,4-6], we presented infinite classes of toric varieties which are set-theoretic complete intersections (i.e., are defined by the vanishing of as many polynomials as their codimension) only in one positive characteristic. In all these cases, the polynomials were, in fact, binomials. This raised the question whether there exists a toric variety $V$ which is defined by $\operatorname{codim} V$ binomial equations in more than one, but not in all positive characteristics. In the present paper we show that the answer is negative if $V$ is simplicial and $\operatorname{codim} V=2$. We give a complete classification of the possible cases in terms of the arithmetic properties of the affine semigroups attached to these varieties. This is an exhaustive study, which includes and completes all previously known results.

Partially supported by the Italian Ministry of Education, University and Research. 


\section{Preliminaries}

Let $K$ be an algebraically closed field, and let $T$ be a finite subset of $\mathbb{Z}^{n}(n \geq 2)$ having cardinality $m>0$, say $T=\left\{\mathbf{t}_{1}, \ldots, \mathbf{t}_{m}\right\}$, where $\mathbf{t}_{i}=\left(t_{i 1}, \ldots, t_{i n}\right)$ for all $i=1, \ldots, m$. We will throughout assume that the set $T$ is not free over $\mathbb{Z}$. Consider the homomorphism of polynomial rings

$$
\phi_{T}: K\left[z_{1}, \ldots, z_{m}\right] \longrightarrow K\left[u_{1}, \ldots, u_{n}\right]
$$

such that

$$
\phi_{T}\left(z_{i}\right)=u_{1}^{t_{i 1}} \cdots u_{n}^{t_{\text {in }}}, \quad \text { for all } i=1, \ldots, m .
$$

The ideal $I_{T}=\operatorname{ker} \phi_{T}$ is generated by all binomials

$$
B_{\alpha_{1}^{-} \cdots \alpha_{m}^{-}}^{\alpha_{1}^{+} \cdots \alpha_{m}^{+}}=z_{1}^{\alpha_{1}^{+}} \cdots z_{m}^{\alpha_{m}^{+}}-z_{1}^{\alpha_{1}^{-}} \cdots z_{m}^{\alpha_{m}^{-}}
$$

where $\alpha_{i}^{+}, \alpha_{i}^{-}$are nonnegative integers (not all zero) such that

$$
\alpha_{1}^{+} \mathbf{t}_{1}+\cdots+\alpha_{m}^{+} \mathbf{t}_{m}=\alpha_{1}^{-} \mathbf{t}_{1}+\cdots+\alpha_{m}^{-} \mathbf{t}_{m},
$$

and in fact there is a one-to-one correspondence between the binomials (1) in $I_{T}$ and the semigroup relations (2) in $\mathbb{N} T$. The ideal $I_{T}$ is the defining ideal of the variety $V_{T} \subset K^{m}$ parameterized by

$$
z_{1}=u_{1}^{t_{11}} \cdots u_{n}^{t_{1 n}}, \quad \cdots, \quad z_{m}=u_{1}^{t_{m 1}} \cdots u_{n}^{t_{m n}},
$$

which is an affine toric variety. If all sums $\sum_{j=1}^{n} t_{i j}$, for $i=1, \ldots, m$, are equal, then this parameterization is called homogeneous and it defines a projective toric variety in $\mathbb{P}^{m-1}$. The dimension of $V_{T}$ is equal to the rank of the Abelian group $\mathbb{Z} T$ if $V$ is affine, it is one less if $V$ is projective.

If $n<m$ and, up to a change of parameters, for all $i=1, \ldots, n$ we have $\mathbf{t}_{i}=c \mathbf{e}_{i}$ with $c$ a positive integer and $\mathbf{e}_{i}$ the $i$ th element of the canonical basis of $\mathbb{Z}^{n}$, then $V$ is called simplicial, and we have $\operatorname{dim} V=n$ if $V$ is affine, $\operatorname{dim} V=n-1$ if $V$ is projective. In this case, whenever $t_{i j} \neq 0$ for all $i=n+1, \ldots, m$ and all $j=1, \ldots, n$, the parameterization of $V$ is called full. A simplicial toric variety of dimension 1 is called a monomial curve: we have an affine monomial curve for $n=1$ and a projective monomial curve if the parameterization is homogeneous and $n=2$; in both cases the parameterization is obviously full. Set codim $V_{T}=r$. Then we say that $V_{T}$ is a (binomial) set-theoretic complete intersection if there are (binomials) $F_{1}, \ldots, F_{r} \in K\left[z_{1}, \ldots, z_{m}\right]$ such that

$$
I_{T}=\sqrt{\left(F_{1}, \ldots, F_{r}\right)}
$$

which, by Hilbert's Nullstellensatz, is equivalent to stating that $V_{T}$ is defined by the following system of $r$ (binomial) equations:

$$
F_{1}=\cdots=F_{r}=0 .
$$


According to the above description, for the variety $V_{T}$ the property of being a complete intersection (i.e., the equality between the minimal number of generators of $I_{T}$ and codim $V_{T}$ ) only depends on $T$, and is thus independent of the ground field $K$. The (binomial) set-theoretic complete intersection property, however, depends, in general, on char $K$. Barile, Morales and Thoma proved the following result.

Proposition 1.1 ([8, Theorem 4]). If char $K=0$, then $V_{T}$ is a binomial set-theoretic complete intersection if and only if it is a complete intersection.

Remark 1.2. There are many examples of affine or projective monomial curves which are not complete intersections, but are set-theoretic complete intersections in characteristic zero: some of them can be found in [9-11,14,17,18,20-22]. This fact, together with Proposition 1.1, shows that being a set-theoretic complete intersection is, in general, a strictly weaker condition than being a binomial set-theoretic complete intersection. We do not know, however, whether this is true in positive characteristics.

The sets $T$ for which the variety $V_{T}$ is a complete intersection have been completely characterized by means of the following notions, introduced by Rosales [19].

Definition 1.3. Let $T_{1}$ and $T_{2}$ be nonempty subsets of $T$ such that $T=T_{1} \cup T_{2}$ and $T_{1} \cap T_{2}=\emptyset$. Then $T$ is called a gluing of $T_{1}$ and $T_{2}$ if there is a nonzero element $\mathbf{w} \in \mathbb{N}^{n}$ such that $\mathbb{Z} T_{1} \cap \mathbb{Z} T_{2}=\mathbb{Z} \mathbf{w}$ and $\mathbf{w} \in \mathbb{N} T_{1} \cap \mathbb{N} T_{2}$.

Definition 1.4. The set $T$ is called a complete intersection if $T$ is the gluing of $T_{1}$ and $T_{2}$, where each of the subsets $T_{1}, T_{2}$ is a complete intersection or generates a free Abelian semigroup.

The next result is due to Fischer, Morris, and Shapiro [12].

Proposition 1.5. The variety $V_{T}$ is a complete intersection if and only if $T$ is the gluing of two subsets $T_{1}$ and $T_{2}$ which are complete intersections.

Proposition 1.1 is false in positive characteristic: here a necessary and sufficient condition for being a binomial set-theoretic complete intersection can be formulated in terms of an arithmetic property of the set $T$ and of the affine semigroup $\mathbb{N} T$, which is derived from the ones given in Definitions 1.3 and 1.4 and was introduced in [8, pp. 1894-1895], by means of the following two definitions.

Definition 1.6. Let $p$ be a prime number and let $T_{1}$ and $T_{2}$ be nonempty subsets of $T$ such that $T=T_{1} \cup T_{2}$ and $T_{1} \cap T_{2}=\emptyset$. Then $T$ is called a p-gluing of $T_{1}$ and $T_{2}$ if there are a nonnegative integer $k$ and a nonzero element $\mathbf{w} \in \mathbb{Z}^{n}$ such that $\mathbb{Z} T_{1} \cap \mathbb{Z} T_{2}=\mathbb{Z} \mathbf{w}$ and $p^{k} \mathbf{w} \in \mathbb{N} T_{1} \cap \mathbb{N} T_{2}$.

Definition 1.7. The set $T$ is called completely $p$-glued if $T$ is the $p$-gluing of $T_{1}$ and $T_{2}$, where each of the subsets $T_{1}, T_{2}$ is completely $p$-glued or generates a free Abelian semigroup. 
Remark 1.8. The comparison of the above definitions yields the following:

(i) If $T$ is the gluing of two nonempty subsets $T_{1}$ and $T_{2}$, then it is the $p$-gluing of $T_{1}$ and $T_{2}$ for all primes $p$.

(ii) If $T$ is a complete intersection, then it is completely $p$-glued for all primes $p$.

Proposition 1.9 ([8, Theorem 5]). If char $K=p$, then $V_{T}$ is a binomial set-theoretic complete intersection if and only if $T$ is completely p-glued.

The papers $[2,4-6]$ present classes of toric varieties that fulfil this property in exactly one positive characteristic $p$, whereas those described in [3], and some of those contained in [5], do not fulfil it for any $p$. In all other known cases the property holds for all $p$. We recall two general results in this direction.

Proposition 1.10 ([7, Theorem 1]). Every simplicial toric variety with a full parameterization is a binomial set-theoretic intersection in all positive characteristics.

This proposition includes the following one as a special case.

Proposition 1.11 ([15, Corollary]). Every projective monomial curve is a binomial set-theoretic complete intersection in all positive characteristics.

So far no toric variety is known which is a binomial set-theoretic complete intersection in more than one, but not in all positive characteristics. The aim of this paper is to show that no such examples exist among the simplicial toric varieties of codimension 2. In sections $3-5$ we will give a complete classification of $p$-gluings for affine simplicial toric varieties of codimension 2 . In section 4 , in particular, we will introduce an arithmetic invariant which allows us to distinguish between the possible cases.

In section 2 we will preliminarily clarify the role of Propositions 1.10 and 1.11 in the general arithmetical framework of completely $p$-glued sets. We will also prove the following additional result.

Proposition 1.12. Every affine simplicial toric variety of dimension at most 2 is a binomial set-theoretic complete intersection in all positive characteristics.

\section{Some known results revisited}

In the sequel, given any $\mathbf{v} \in \mathbb{Z}^{n}$, we will denote by $v_{i}$ its $i$-th component, for $1 \leq i \leq n$. We will consider the varieties $V_{T}$, where $T=\left\{\mathbf{a}, \mathbf{b}, c \mathbf{e}_{1}, \ldots, c \mathbf{e}_{n}\right\} \subset \mathbb{Z}^{n}$, and $c$ is any positive integer. This class consists of all affine (or projective) simplicial toric varieties of codimension 2 in $K^{n+2}$ (or in $\mathbb{P}^{n+1}$ ). It is not restrictive to assume that, for all $i=1, \ldots, n$, we have $a_{i} \neq 0$ or $b_{i} \neq 0$. We introduce the following two partitions 
of $T$ :

$$
\begin{array}{ll}
\tilde{T}_{1}=\{\mathbf{a}\}, & \tilde{T}_{2}=\left\{\mathbf{b}, c \mathbf{e}_{1}, \ldots, c \mathbf{e}_{n}\right\}, \\
\tilde{\tilde{T}}_{1}=\{\mathbf{b}\}, & \tilde{\tilde{T}}_{2}=\left\{\mathbf{a}, c \mathbf{e}_{1}, \ldots, c \mathbf{e}_{n}\right\} .
\end{array}
$$

For all $\mathbf{v} \in \mathbb{Z}^{n}$ we will set $\operatorname{supp} \mathbf{v}=\left\{i \mid v_{i} \neq 0\right\}$. The content of the next two propositions is not totally new, since they partly consist of results collected from other sources. Nevertheless, we find it useful and interesting to re-propose these statements here in a unified form, which emphasizes the role of $p$-gluings. The proof of the next proposition under the assumption (i) is hinted at in [8, Example 1].

Proposition 2.1. Suppose that either

(i) $\operatorname{supp} \mathbf{b} \subset \operatorname{supp} \mathbf{a}$, or

(ii) $\operatorname{supp} \mathbf{b}=\{1, \ldots, n\} \backslash \operatorname{supp} \mathbf{a}$.

Then $T$ is the p-gluing of $\tilde{T}_{1}$ and $\tilde{T}_{2}$ for all primes $p$. (If $\mathbf{a}$ and $\mathbf{b}$ are exchanged, then $T$ is the p-gluing of $\tilde{\tilde{T}}_{1}$ and $\tilde{\tilde{T}}_{2}$ for all primes $p$.)

Proof. Suppose that (i) holds. Since

$$
c \mathbf{a}=\sum_{i=1}^{n} a_{i} c \mathbf{e}_{i} \in \mathbb{Z} \tilde{T}_{2},
$$

the number

$$
\alpha=\operatorname{gcd}\left\{\lambda \in \mathbb{Z} \mid \lambda \mathbf{a} \in \mathbb{Z} \tilde{T}_{2}\right\}
$$

is a well-defined positive integer. Moreover

$$
\mathbb{Z} \tilde{T}_{1} \cap \mathbb{Z} \tilde{T}_{2}=\mathbb{Z} \alpha \mathbf{a} .
$$

We have that, for some integers $\beta, \gamma_{1}, \ldots, \gamma_{n}$,

$$
\alpha \mathbf{a}=\beta \mathbf{b}+\sum_{i=1}^{n} \gamma_{i} c \mathbf{e}_{i},
$$

i.e., for all $i=1, \ldots, n$ we have the following equality, where either side is a natural number,

$$
\alpha a_{i}=\beta b_{i}+\gamma_{i} c .
$$

We have to show that under the given assumption, for all primes $p$ there is a nonnegative integer $k$ such that

$$
p^{k} \alpha \mathbf{a} \in \mathbb{N} \tilde{T}_{2} .
$$


Suppose that $\operatorname{supp} \mathbf{b} \subset \operatorname{supp} \mathbf{a}$. Then, for all $i \in \operatorname{supp} \mathbf{b}$, in (4) we have $\alpha a_{i}>0$, whence $-\frac{\gamma_{i}}{b_{i}}<\frac{\beta}{c}$. Set

$$
\gamma=\max _{i \in \operatorname{supp} \mathbf{b}}-\frac{\gamma_{i}}{b_{i}} .
$$

Then $\gamma<\frac{\beta}{c}$. Let $p$ be any prime number. We can choose a sufficiently large positive integer $k$ so as to have

$$
p^{k} \gamma<d<p^{k} \frac{\beta}{c}
$$

for some integer $d$. Set

$$
\beta^{\prime}=p^{k} \beta-d c,
$$

and, for all $i=1, \ldots, n$,

$$
\gamma_{i}^{\prime}=p^{k} \gamma_{i}+d b_{i} .
$$

Then (7) implies that $\beta^{\prime}>0$ and, moreover, from (6) and (7), for all $i \in \operatorname{supp} \mathbf{b}$, we get

$$
p^{k} \frac{\gamma_{i}}{b_{i}} \geq-p^{k} \gamma>-d,
$$

which implies that $\gamma_{i}^{\prime}>0$. If $i \notin \operatorname{supp} \mathbf{b}$, then from (4) we deduce that $\alpha a_{i}=\gamma_{i} c$, which gives us $\gamma_{i}>0$, so that $\gamma_{i}^{\prime}=p^{k} \gamma_{i}>0$. Hence

$$
p^{k} \alpha \mathbf{a}=\beta^{\prime} \mathbf{b}+\sum_{i=1}^{n} \gamma_{i}^{\prime} c \mathbf{e}_{i},
$$

so that (5) is true.

Now suppose that (ii) holds. For all $i \in \operatorname{supp} \mathbf{a}$ we have $a_{i}>0$ and $b_{i}=0$. Thus, in view of (4) we also have $\alpha a_{i}=\gamma_{i} c$, where $\gamma_{i}>0$. Therefore

$$
\alpha \mathbf{a}=\sum_{i \in \operatorname{supp} \mathbf{a}} \gamma_{i} \mathbf{e}_{i} \in \mathbb{N} \tilde{T}_{2},
$$

so that (5) is fulfilled for $k=0$. This completes the proof.

Proposition 2.2. $\quad$ (i) If $T$ is the gluing of $\tilde{T}_{1}$ and $\tilde{T}_{2}$ (or of $\tilde{\tilde{T}}_{1}$ and $\tilde{\tilde{T}}_{2}$ ), then $T$ is a complete intersection.

(ii) Let $p$ be a prime. If $T$ is the p-gluing of $\tilde{T}_{1}$ and $\tilde{T}_{2}$ (or of $\tilde{\tilde{T}}_{1}$ and $\tilde{\tilde{T}}_{2}$ ), then $T$ is completely p-glued.

Proof. We show the claim for $\tilde{T}_{1}$ and $\tilde{T}_{2}$. In view of Remark 1.8 (i), it suffices to show that $\tilde{T}_{2}$ is the gluing of $\tilde{T}_{21}=\{\mathbf{b}\}$ and $\tilde{T}_{22}=\left\{c \mathbf{e}_{1}, \ldots, c \mathbf{e}_{n}\right\}$ : in fact, $\mathbb{N} \tilde{T}_{21}$ and $\mathbb{N} \tilde{T}_{22}$ are both free Abelian semigroups. Now, if $\beta=c / \operatorname{gcd}\left(c, b_{1}, \ldots, b_{n}\right)$, then $\mathbb{Z} \beta \mathbf{b}=\mathbb{Z} \tilde{T}_{21} \cap \mathbb{Z} \tilde{T}_{22}$ and $\beta \mathbf{b} \in \mathbb{N} \tilde{T}_{21} \cap \mathbb{N} \tilde{T}_{22}$. The claim for $\tilde{T}_{1}$ and $\tilde{\tilde{T}}_{2}$ can be shown similarly. This completes the proof. 
Corollary 2.3. If $n \leq 2$, then, for all primes $p, T$ is the p-gluing of $\tilde{T}_{1}$ and $\tilde{T}_{2}$, or of $\tilde{\tilde{T}}_{1}$ and $\tilde{\tilde{T}}_{2}$. In particular, it is completely p-glued for all primes $p$.

Proof. The first part of the claim follows immediately from Proposition 2.1, since for $n=2$ one of (i) and (ii) of that proposition must hold. The second part is a consequence of Proposition 2.2 (ii).

Remark 2.4. Proposition 1.10, Proposition 1.11, and Proposition 1.12 follow from Corollary 2.3 and Proposition 1.9.

\section{The main theorem on $p$-gluings}

In this section we prove the crucial technical result. We consider the set $T$ introduced above and assume that it does not fulfil the assumption (i) of Proposition 2.1 with respect to any order of $\mathbf{a}$ and $\mathbf{b}$. We show that the property of being $p$-glued then reduces to the property of being the $p$-gluing of $\tilde{T}_{1}$ and $\tilde{T}_{2}$ (or, equivalently, of $\tilde{\tilde{T}}_{1}$ and $\left.\tilde{\tilde{T}}_{2}\right)$.

Theorem 3.1. Let $p$ be a prime. Suppose that none of $\operatorname{supp} \mathbf{a}$ and $\operatorname{supp} \mathbf{b}$ is contained in the other. If $T$ is the p-gluing of two nonempty subsets $T_{1}$ and $T_{2}$, then $T$ is the p-gluing of $\tilde{T}_{1}$ and $\tilde{T}_{2}$, and of $\tilde{\tilde{T}}_{1}$ and $\tilde{\tilde{T}}_{2}$.

Proof. As a trivial consequence of the first assumption, we have $n \geq 2$, and we may assume that, up to a change of indices, $a_{1} \neq 0, b_{1}=0, a_{2}=0, b_{2} \neq 0$. By the second assumption there is $\mathbf{w} \in \mathbb{Z}^{n}, \mathbf{w} \neq 0$, such that

$$
\mathbb{Z} \mathbf{w}=\mathbb{Z} T_{1} \cap \mathbb{Z} T_{2} \quad \text { and } \quad p^{k} \mathbf{w} \in \mathbb{N} T_{1} \cap \mathbb{N} T_{2}
$$

for some nonnegative integer $k$. Let

$$
T_{1}=\left\{\mathbf{t}_{11}, \ldots, \mathbf{t}_{1 r}\right\}, \quad \text { and } \quad T_{2}=\left\{\mathbf{t}_{21}, \ldots, \mathbf{t}_{2 s}\right\} .
$$

Then we have

$$
\mathbf{w}=\sum_{i=1}^{r} \alpha_{i} \mathbf{t}_{1 i}=\sum_{i=1}^{s} \beta_{i} \mathbf{t}_{2 i}
$$

for some $\alpha_{1}, \ldots, \alpha_{r}, \beta_{1}, \ldots, \beta_{s} \in \mathbb{Z}$, and

$$
p^{k} \mathbf{w}=\sum_{i=1}^{r} \tilde{\alpha}_{i} \mathbf{t}_{1 i}=\sum_{i=1}^{s} \tilde{\beta}_{i} \mathbf{t}_{2 i}
$$

for some $\tilde{\alpha}_{1}, \ldots, \tilde{\alpha}_{r}, \tilde{\beta}_{1}, \ldots, \tilde{\beta}_{s} \in \mathbb{N}$. Since $T_{1} \cup T_{2}=T$, up to exchanging $T_{1}$ and $T_{2}$ we may assume that $\mathbf{a} \in T_{1}$, say $\mathbf{t}_{11}=\mathbf{a}$, so that $\alpha_{1}$ is the coefficient of $\mathbf{a}$ in (9) and $\tilde{\alpha}_{1}$ is the coefficient of $\mathbf{a}$ in (10). We will also adopt the following notation:

$$
I_{1}=\left\{i \mid c \mathbf{e}_{i} \in T_{1}\right\} \quad \text { and } \quad I_{2}=\left\{i \mid c \mathbf{e}_{i} \in T_{2}\right\} .
$$


The theorem will be proven in several steps, through a sequence of intermediate claims.

Claim 1. (i) One of $\mathbf{a}$ and $\mathbf{b}$ appears in (10) with a nonzero coefficient.

(ii) If $\tilde{\alpha}_{1} \neq 0$, then $\alpha_{1} \neq 0$ and $1 \in I_{2}$.

Proof of Claim 1. If $\mathbf{a}$ and $\mathbf{b}$ both appeared in (10) with a zero coefficient, then we would have $\mathbf{w}=0$, because the set $\left\{c \mathbf{e}_{1}, \ldots, c \mathbf{e}_{n}\right\}$ is free, against our assumption on $\mathbf{w}$. This proves (i). We now prove (ii). Suppose that $\tilde{\alpha}_{1} \neq 0$. Then $1 \in I_{2}$, otherwise we could assume that $\mathbf{t}_{12}=c \mathbf{e}_{1}$ and we would deduce that

$$
p^{k} w_{1}=\tilde{\alpha}_{1} a_{1}+\tilde{\alpha}_{2} c=0,
$$

because $b_{1}=0$. Since all elements in the above equality are nonnegative integers, it would follow that $a_{1}=0$, against our assumption. Now suppose for a contradiction that $\alpha_{1}=0$. Then, since $1 \in I_{2}$ and $b_{1}=0$, from (9) we deduce that $w_{1}=0$. But the element

$$
\mathbf{u}=c \mathbf{a}-\sum_{i \in I_{1}} a_{i} c \mathbf{e}_{i}=\sum_{i \in I_{2}} a_{i} c \mathbf{e}_{i}
$$

also belongs to $\mathbb{Z} T_{1} \cap \mathbb{Z} T_{2}$. However, since $u_{1}=a_{1} c \neq 0$, we conclude that $\mathbf{u}$ cannot belong to $\mathbb{Z} \mathbf{w}$, which contradicts (8). This shows that $\alpha_{1} \neq 0$ and completes the proof of Claim 1.

Claim 2. If $1 \in I_{2}$, then

$$
\mathbb{Z} \alpha_{1} \mathbf{a}=\mathbb{Z} \tilde{T}_{1} \cap \mathbb{Z} \tilde{T}_{2},
$$

and $\tilde{\alpha}_{1}=p^{k} \alpha_{1}$

Proof of Claim 2. In view of (9),

$$
\alpha_{1} \mathbf{a} \in \mathbb{Z} \tilde{T}_{1} \cap \mathbb{Z} \tilde{T}_{2} .
$$

This intersection is a subgroup of $\mathbb{Z} \mathbf{a}$, hence it is $\mathbb{Z} \gamma \mathbf{a}$, for some integer $\gamma$ having the same sign as $\alpha_{1}$. Since $\alpha_{1} \mathbf{a} \in \mathbb{Z} \gamma \mathbf{a}$, we also have that $\gamma$ divides $\alpha_{1}$. Since $\gamma \mathbf{a} \in \mathbb{Z} \tilde{T}_{2}$, the element $\gamma \mathbf{a}=\gamma \mathbf{t}_{11}$ can be expressed as a linear combination, with integer coefficients, of $\mathbf{b}, \mathbf{e}_{1}, \ldots, \mathbf{e}_{n}$, i.e., of $\mathbf{t}_{12}, \ldots, \mathbf{t}_{1 r}, \mathbf{t}_{21}, \ldots, \mathbf{t}_{2 s}$. We thus have an equality

$$
\sum_{i=1}^{r} \alpha_{i}^{\prime} \mathbf{t}_{1 i}=\sum_{i=1}^{s} \beta_{i}^{\prime} \mathbf{t}_{2 i}
$$

for $\alpha_{1}^{\prime}=\gamma$ and some $\alpha_{2}^{\prime}, \ldots, \alpha_{r}^{\prime}, \beta_{1}^{\prime}, \ldots, \beta_{s}^{\prime} \in \mathbb{Z}$. The element on either side of (12) belongs to $\mathbb{Z} T_{1} \cap \mathbb{Z} T_{2}$, hence it is equal to $\lambda \mathbf{w}$ for some $\lambda \in \mathbb{Z}$. Thus (12) implies that

$$
\lambda w_{1}=\gamma a_{1},
$$


because $1 \in I_{2}$ and $b_{1}=0$. On the other hand, (9) yields

$$
w_{1}=\alpha_{1} a_{1},
$$

but then, replacing (14) in (13), we get $\lambda \alpha_{1}=\gamma$, so that $\alpha_{1}$ divides $\gamma$. We thus conclude that $\alpha_{1}$ and $\gamma$ are associate integers of the same sign, i.e., $\alpha_{1}=\gamma$. Furthermore, equality (10) gives us $p^{k} w_{1}=\tilde{\alpha}_{1} a_{1}$, from which, in view of (14), we deduce that $\tilde{\alpha}_{1}=p^{k} \alpha_{1}$, as required. This completes the proof of Claim 2.

Claim 3. If $\tilde{\alpha}_{1} \neq 0$, then $\mathbf{b}$ appears in (10) with coefficient zero.

Proof of Claim 3. Suppose for a contradiction that, under the given hypothesis, b appears in (10) with a nonzero coefficient. There is $j \in\{1,2\}$ such that $\mathbf{b} \in T_{j}$. Therefore, replacing a with $\mathbf{b}, \mathbf{e}_{1}$ with $\mathbf{e}_{2}$, and $T_{1}$ with $T_{j}$, Claim 1 (ii) allows us to conclude that $c \mathbf{e}_{2} \notin T_{j}$. Then

$$
\mathbf{v}=c \mathbf{b}-\sum_{i \in I_{j}} b_{i} c \mathbf{e}_{i}=\sum_{i \notin I_{j}} b_{i} c \mathbf{e}_{i} \in \mathbb{Z} T_{1} \cap \mathbb{Z} T_{2},
$$

and $v_{1}=0$, because $b_{1}=0$, whereas $v_{2}=b_{2} c \neq 0$. But then $\mathbf{v}$ and the element $\mathbf{u}$ from (11) cannot both belong to $\mathbb{Z} \mathbf{w}$, which contradicts (8). This proves Claim 3.

Claim 4. If $\tilde{\alpha}_{1} \neq 0$, then $T$ is the p-gluing of $\tilde{T}_{1}$ and $\tilde{T}_{2}$.

Proof of Claim 4. From (10) we get, in view of Claim 1 (ii), Claim 2, and Claim 3,

$$
\tilde{\alpha}_{1} \mathbf{a}=p^{k} \alpha_{1} \mathbf{a} \in \mathbb{Z}\left\{c \mathbf{e}_{1}, \ldots, c \mathbf{e}_{n}\right\},
$$

which implies that

$$
p^{k}\left|\alpha_{1}\right| \mathbf{a} \in \mathbb{N}\left\{c \mathbf{e}_{1}, \ldots, c \mathbf{e}_{n}\right\} \subset \mathbb{N} \tilde{T}_{2} .
$$

On the other hand, by Claim 2,

$$
\mathbb{Z}\left|\alpha_{1}\right| \mathbf{a}=\mathbb{Z} \tilde{T}_{1} \cap \mathbb{Z} \tilde{T}_{2},
$$

which, together with (15), implies that $T$ is the $p$-gluing of $\tilde{T}_{1}$ and $\tilde{T}_{2}$. This proves Claim 4.

Claim 5. T is the p-gluing of $\tilde{\tilde{T}}_{1}$ and $\tilde{\tilde{T}}_{2}$.

Proof of Claim 5. According to Claim 1 (i), we have that either $\mathbf{a}$ or $\mathbf{b}$ appears in (10) with a nonzero coefficient. In the latter case we have that $T$ is the $p$-gluing of $\tilde{\tilde{T}}_{1}$ and $\tilde{\tilde{T}}_{2}$ : this follows from Claim 4 , after exchanging a and $\mathbf{b}$. So assume that the former case occurs, i.e., that $\tilde{\alpha}_{1} \neq 0$. Then, by Claim 1 (ii), $1 \in I_{2}$. Let $\beta^{\prime} \geq 0$ be such that

$$
\mathbb{Z} \beta^{\prime} \mathbf{b}=\mathbb{Z} \tilde{\tilde{T}}_{1} \cap \mathbb{Z} \tilde{\tilde{T}}_{2}
$$


Then, for some integers $\alpha^{\prime}, \gamma_{1}^{\prime}, \ldots, \gamma_{n}^{\prime}$,

$$
\beta^{\prime} \mathbf{b}=\alpha^{\prime} \mathbf{a}+\sum_{i=1}^{n} \gamma_{i}^{\prime} \mathbf{e}_{i},
$$

so that, $\alpha^{\prime} \mathbf{a} \in \mathbb{Z} \tilde{T}_{1} \cap \mathbb{Z} \tilde{T}_{2}$ and therefore, in view of Claim 2, $\alpha_{1}$ divides $\alpha^{\prime}$. Hence (17) implies that

$$
\beta^{\prime} \mathbf{b} \in \mathbb{Z}\left\{\alpha_{1} \mathbf{a}, c \mathbf{e}_{1}, \ldots, c \mathbf{e}_{n}\right\},
$$

whence

$$
p^{k} \beta^{\prime} \mathbf{b} \in \mathbb{Z}\left\{p^{k} \alpha_{1} \mathbf{a}, c \mathbf{e}_{1}, \ldots, c \mathbf{e}_{n}\right\} \subset \mathbb{Z}\left\{c \mathbf{e}_{1}, \ldots, c \mathbf{e}_{n}\right\},
$$

where the inclusion follows from (15). Therefore $p^{k} \beta^{\prime} \mathbf{b} \in \mathbb{N}\left\{c \mathbf{e}_{1}, \ldots, c \mathbf{e}_{n}\right\} \subset \mathbb{N} \tilde{\tilde{T}}_{2}$. This, together with (16), shows that $T$ is the $p$-gluing of $\tilde{\tilde{T}}_{1}$ and $\tilde{\tilde{T}}_{2}$. This completes the proof of Claim 5 .

We can now conclude the proof of the theorem. By Claim 5, $T$ is the $p$-gluing of $\tilde{\tilde{T}}_{1}$ and $\tilde{\tilde{T}}_{2}$. The argumentation developed above, with $\mathbf{a}$ and $\mathbf{b}$ exchanged, allow us to conclude that $T$ is the $p$-gluing of $\tilde{T}_{1}$ and $\tilde{T}_{2}$ as well. This completes the proof of the theorem.

Lemma 3.2. If $T$ is the p-gluing of two nonempty subsets $T_{1}$ and $T_{2}$ for two different primes $p$, then it is the $p$-gluing of $T_{1}$ and $T_{2}$ for all primes $p$.

Proof. If $T$ is the $p$-gluing and the $p^{\prime}$-gluing of $T_{1}$ and $T_{2}$, where $p$ and $p^{\prime}$ are distinct primes, then there is $\mathbf{w} \in \mathbb{Z}^{n}$ such that $\mathbb{Z} \mathbf{w}=\mathbb{Z} T_{1} \cap \mathbb{Z} T_{2}$ and

$$
p^{k} \mathbf{w}, p^{\prime k^{\prime}} \mathbf{w} \in \mathbb{N} T_{1} \cap \mathbb{N} T_{2},
$$

for some nonnegative integers $k, k^{\prime}$. Let $q$ be a prime. Then there are $a, b, \ell \in \mathbb{N}$ such that

$$
q^{\ell}=a p^{k}+b p^{\prime k^{\prime}},
$$

so that, by (18),

$$
q^{\ell} \mathbf{w} \in \mathbb{N} T_{1} \cap \mathbb{N} T_{2} .
$$

This proves that $T$ is the $q$-gluing of $T_{1}$ and $T_{2}$, which completes the proof.

Proposition 2.1, Theorem 3.1, and Lemma 3.2 imply the following result.

Corollary 3.3. If $T$ is completely p-glued for two distinct primes $p$, then it is completely p-glued for all primes $p$.

The converse of statement (ii) of Remark 1.8 is not true: Propositions 1.10 and 1.11 provide infinitely many examples of sets $T$ which are completely $p$-glued for all primes $p$, but are not complete intersections. We, however, have the following result. 
Corollary 3.4. Suppose that neither of supp $\mathbf{a}$ and $\operatorname{supp} \mathbf{b}$ is contained in the other. Then $T$ is completely p-glued for all primes $p$ if and only if $T$ is a complete intersection.

Proof. We just need to prove the only if part. Suppose that $T$ is completely $p$-glued and $q$-glued, where $p$ and $q$ are distinct primes. By virtue of Theorem 3.1, $T$ is the $p$-gluing and the $q$-gluing of $\tilde{T}_{1}$ and $\tilde{T}_{2}$, so that, for some nonzero $\alpha \in \mathbb{N}$, we have

$$
\mathbb{Z} \alpha \mathbf{a}=\mathbb{Z} \tilde{T}_{1} \cap \mathbb{Z} \tilde{T}_{2},
$$

and $p^{k} \alpha \mathbf{a} \in \mathbb{N} \tilde{T}_{2}, q^{k} \alpha \mathbf{a} \in \mathbb{N} \tilde{T}_{2}$, for some nonnegative integer $k$. Since supp $\mathbf{b} \not \subset \operatorname{supp} \mathbf{a}$, this implies that $p^{k} \alpha \mathbf{a}, q^{k} \alpha \mathbf{a} \in \mathbb{N}\left\{c \mathbf{e}_{1}, \ldots, c \mathbf{e}_{n}\right\}$. Let $g=c / \operatorname{gcd}\left(c, a_{1}, \ldots, a_{n}\right)$. Then $\mathbb{N} g \mathbf{a}=\mathbb{N} \mathbf{a} \cap \mathbb{N}\left\{c \mathbf{e}_{1}, \ldots, c \mathbf{e}_{n}\right\}$. Thus $g$ divides both $p^{k} \alpha$ and $q^{k} \alpha$, so that $g$ divides $\alpha$. But then $\alpha \mathbf{a} \in \mathbb{N}\left\{c \mathbf{e}_{1}, \ldots, c \mathbf{e}_{n}\right\}$, whence

$$
\alpha \mathbf{a} \in \mathbb{N} \tilde{T}_{1} \cap \mathbb{N} \tilde{T}_{2} .
$$

Relations (19) and (20) show that $T$ is the gluing of $\tilde{T}_{1}$ and $\tilde{T}_{2}$. In view of Proposition 2.2 (i), it follows that $T$ is a complete intersection. This completes the proof.

Proposition 2.1, Theorem 3.1 and Corollary 3.3 imply the following.

Corollary 3.5. The set $T$ is completely p-glued

(i) for exactly one prime $p$, or

(ii) for every prime $p$, or

(iii) for no prime $p$.

Moreover, if $T$ is completely p-glued, then it is the p-gluing of $\tilde{T}_{1}$ and $\tilde{T}_{2}$ (or of $\tilde{\tilde{T}}_{1}$ and $\left.\tilde{\tilde{T}}_{2}\right)$.

\section{A computational point of view}

In this section we provide a complete arithmetic characterization of the sets $T$ fulfilling each of the cases listed in Corollary 3.5 under the assumption that neither of supp a and supp $\mathbf{b}$ is contained in the other. Our first step is to derive a closed formula for the number $\alpha$ in (3). Up to dividing the elements of $T$ by their greatest common divisor $\delta$ (which corresponds to a change of parameters for $V_{T}$, in which, for all $i=1, \ldots, n$, $u_{i}^{c}, u_{i}^{a_{i}}$, and $u_{i}^{b_{i}}$ are replaced by $u_{i}^{c / \delta}, u_{i}^{a_{i} / \delta}$, and $u_{i}^{b_{i} / \delta}$, respectively), we may assume that

$$
\operatorname{gcd}\left(c, a_{1}, \ldots, a_{n}, b_{1}, \ldots, b_{n}\right)=1
$$


For all $\lambda \in \mathbb{N}$ consider the $n \times(n+2)$-matrix with integer entries

$$
A_{\lambda}=\left(\begin{array}{cccccc}
\lambda a_{1} & b_{1} & c & 0 & \cdots & 0 \\
\vdots & \vdots & 0 & \ddots & & \vdots \\
\vdots & \vdots & \vdots & & \ddots & 0 \\
\lambda a_{n} & b_{n} & 0 & \cdots & 0 & c
\end{array}\right)
$$

and the $n \times(n+1)$-submatrix

$$
A^{\prime}=\left(\begin{array}{ccccc}
b_{1} & c & 0 & \cdots & 0 \\
\vdots & 0 & \ddots & & \vdots \\
\vdots & \vdots & & \ddots & 0 \\
b_{n} & 0 & \cdots & 0 & c
\end{array}\right) .
$$

For all $\lambda \in \mathbb{N}$ let $g_{\lambda}$ be the greatest common divisor of the $n$-minors of $A_{\lambda}$ and let $g^{\prime}$ be the greatest common divisor of the $n$-minors of $A^{\prime}$. Moreover, set

$$
\bar{g}=\underset{1 \leq i<j \leq n}{\operatorname{gcd}}\left(c^{n-1} a_{i}, c^{n-2}\left(a_{i} b_{j}-a_{j} b_{i}\right)\right) .
$$

Then

$$
g_{\lambda}=\operatorname{gcd}\left(g^{\prime}, \lambda \bar{g}\right) .
$$

If we apply the solvability criterion for diophantine systems given in [13] to $A^{\prime} \mathbf{x}=\lambda \mathbf{a}$, we conclude that $\lambda \mathbf{a} \in \mathbb{Z} \tilde{T}_{2}$ if and only if $g^{\prime}=g_{\lambda}$, i.e., if and only if $g^{\prime}$ divides $\lambda \bar{g}$, which is the case if and only if $\lambda$ is a multiple of

$$
\alpha=\frac{g^{\prime}}{\operatorname{gcd}\left(g^{\prime}, \bar{g}\right)} .
$$

Note that

$$
g^{\prime}=\underset{1 \leq i \leq n}{\operatorname{gcd}}\left(c^{n}, c^{n-1} b_{i}\right)=c^{n-1} \underset{1 \leq i \leq n}{\operatorname{gcd}}\left(c, b_{i}\right)=c^{n-2} \underset{1 \leq i \leq n}{\operatorname{gcd}}\left(c^{2}, c b_{i}\right),
$$

and

$$
\bar{g}=c^{n-2} \underset{1 \leq i<j \leq n}{\operatorname{gcd}}\left(c a_{i}, a_{i} b_{j}-a_{j} b_{i}\right)
$$

so that

$$
\begin{aligned}
& \operatorname{gcd}\left(g^{\prime}, \bar{g}\right)=c^{n-2} \underset{1 \leq i<j \leq n}{\operatorname{gcd}}\left(c^{2}, c a_{i}, c b_{i}, a_{i} b_{j}-a_{j} b_{i}\right) \\
& =c^{n-2} \underset{1 \leq i<j \leq n}{\operatorname{gcd}}\left(c \operatorname{gcd}\left(c, a_{i}, b_{i}\right), a_{i} b_{j}-a_{j} b_{i}\right) \\
& =c^{n-2} \underset{1 \leq i<j \leq n}{\operatorname{gcd}}\left(c, a_{i} b_{j}-a_{j} b_{i}\right) \text {, }
\end{aligned}
$$


where the last equality follows from (21). Finally, from (22), (23), and (24) we deduce that

$$
\alpha=\frac{c \operatorname{gcd}_{1 \leq i \leq n}\left(c, b_{i}\right)}{\operatorname{gcd}_{1 \leq i \leq n}\left(c, a_{i} b_{j}-a_{j} b_{i}\right)},
$$

which is the sought closed formula.

We are now ready to prove the main result of this section, which completes Corollary 2 in [7].

Proposition 4.1. Suppose that neither of $\operatorname{supp} \mathbf{a}$ and $\operatorname{supp} \mathbf{b}$ is contained in the other. Let $c^{\prime}=\operatorname{gcd}_{1 \leq i<j \leq n}\left(c, a_{i} b_{j}-a_{j} b_{i}\right)$, and set

$$
g(T)=\frac{c^{\prime}}{\operatorname{gcd}_{1 \leq i \leq n}\left(c^{\prime}, a_{i}\right) \operatorname{gcd}_{1 \leq i \leq n}\left(c^{\prime}, b_{i}\right)} .
$$

Then $T$ is completely p-glued

(i) for exactly one prime $p$, if and only if $g(T)$ is a positive power of $p$;

(ii) for every prime $p$, if and only if $g(T)$ is equal to 1;

(iii) for no prime $p$, if and only if $g(T)$ has two distinct prime divisors.

Case (ii) occurs if and only if $T$ is a complete intersection.

Proof. We preliminarily note that the last claim is a consequence of Corollary 3.4. Let $p$ be any prime. By virtue of Theorem 3.1 and Proposition 2.2 (ii), $T$ is completely $p$-glued if and only if it is the $p$-gluing of $\tilde{T}_{1}$ and $\tilde{T}_{2}$. This occurs if and only if $p^{k} \alpha \mathbf{a} \in \mathbb{N} \tilde{T}_{2}$, which is equivalent to

$$
p^{k} \alpha \mathbf{a} \in \mathbb{N}\left\{c \mathbf{e}_{1}, \ldots, c \mathbf{e}_{n}\right\},
$$

for some nonnegative integer $k$, where $\alpha$ is the positive integer defined in (3) and given by the formula (25). Now (26) is true if and only if $p^{k}$ is a multiple of

$$
\omega=\frac{c}{\operatorname{gcd}_{1 \leq i \leq n}\left(c, \alpha a_{i}\right)}=\frac{c}{\operatorname{gcd}\left(c, \alpha \operatorname{gcd}_{1 \leq i \leq n}\left(a_{i}\right)\right)} .
$$

Thus (26) is true for exactly one prime $p$, for every prime $p$, or for no prime $p$ if $\omega$ is a positive power of $p$, is equal to 1 , or has two distinct prime divisors, respectively. 
In view of (25) we have

$$
\begin{aligned}
\omega & =\frac{c}{\operatorname{gcd}\left(c, \frac{c}{c^{\prime}} \operatorname{gcd}_{1 \leq i \leq n}\left(c, b_{i}\right) \operatorname{gcd}_{1 \leq i \leq n}\left(a_{i}\right)\right)} \\
& =\frac{c}{\frac{c}{c^{\prime}} \operatorname{gcd}\left(c^{\prime}, \operatorname{gcd}_{1 \leq i \leq n}\left(c, b_{i}\right) \operatorname{gcd}_{1 \leq i \leq n}\left(a_{i}\right)\right)} \\
& =\frac{c^{\prime}}{\operatorname{gcd}\left(c^{\prime}, \operatorname{gcd}_{1 \leq i \leq n}\left(c^{\prime}, b_{i}\right) \operatorname{gcd}_{1 \leq i \leq n}\left(a_{i}\right)\right)} \\
& =\frac{c^{\prime}}{\operatorname{gcd}\left(c^{\prime}, \operatorname{gcd}_{1 \leq i \leq n}\left(c^{\prime}, b_{i}\right) \operatorname{gcd}_{1 \leq i \leq n}\left(c^{\prime}, a_{i}\right)\right)} \\
& =\frac{c^{\prime}}{\operatorname{gcd}_{1 \leq i \leq n}\left(c^{\prime}, b_{i}\right) \operatorname{gcd}_{1 \leq i \leq n}\left(c^{\prime}, a_{i}\right)},
\end{aligned}
$$

where the last equality follows from the fact that, as a consequence of (21), $\operatorname{gcd}\left(c^{\prime}, a_{1}, \ldots, a_{n}, b_{1}, \ldots, b_{n}\right)=1$. This shows that $\omega=g(T)$ and completes the proof.

Remark 4.2. Since $g(T)$ is a divisor of $c$, case (iii) of Proposition 4.1 cannot hold whenever $c$ is a prime power. From this we deduce the well-known fact that $T$ is always completely $p$-glued if $c=p^{r}$ for some nonnegative integer $r$ (this statement holds for simplicial toric varieties of any codimension: for a proof see, e.g., [5, Proposition 2]). In [6] we considered the case where $c=p$. There, in Theorem 2.1, we gave a different characterization of case (i) in form of four arithmetic conditions.

\section{Applications to set-theoretic complete intersections}

We now use the results on $p$-gluings proven in section 3 for giving a complete characterization of simplicial toric varieties of codimension 2 with respect to the property of being a binomial set-theoretic complete intersection. The (affine or projective) toric variety $V_{T}$ attached to the set $T$ considered in the previous sections admits the following parameterization:

$$
x_{1}=u_{1}^{c}, \quad \ldots, \quad x_{n}=u_{n}^{c}, \quad y_{1}=u_{1}^{a_{1}} \cdots u_{n}^{a_{n}}, \quad y_{2}=u_{1}^{b_{1}} \cdots u_{n}^{b_{n}} .
$$

As an immediate consequence of Proposition 1.9 and Corollary 3.5 we have the following.

Corollary 5.1. A simplicial toric variety of codimension 2 is a binomial set-theoretic complete intersection in characteristic $p$ either

(i) for exactly one prime $p$, or

(ii) for every prime $p$, or 
(iii) for no prime $p$.

Example 5.2. Suppose that $T$ is the $p$-gluing of $\tilde{T}_{1}$ and $\tilde{T}_{2}$, with $\mathbb{Z} \tilde{T}_{1} \cap \mathbb{Z} \tilde{T}_{2}=\mathbb{Z} \alpha \mathbf{a}$ and $p^{k} \alpha \mathbf{a}=\beta \mathbf{b}+\sum_{i=1}^{n} \beta_{i} \mathbf{e}_{i}$, where $k, \beta, \beta_{1} \ldots \beta_{n} \in \mathbb{N}$. Then the construction performed in the proof of [8, Theorem 5] shows that $V_{T}$ is a set-theoretic complete intersection defined by the vanishing of the following two binomials:

$$
F_{1}=y_{1}^{p^{k} \alpha}-y_{2}^{\beta} x_{1}^{\beta_{1}} \cdots x_{n}^{\beta_{n}}, \quad F_{2}=y_{2}^{\gamma}-x_{1}^{\delta_{1}} \cdots x_{n}^{\delta_{n}} .
$$

Here we have set $\gamma=\frac{c}{\operatorname{gcd}_{1 \leq j \leq n}\left(c, b_{j}\right)}$ and $\delta_{i}=\frac{b_{i}}{\operatorname{gcd}_{1 \leq j \leq n}\left(c, b_{j}\right)}$ for all $i=1, \ldots, n$.

Remark 5.3. According to [6, Theorem 2.1], if $c=p$, then $V_{T}$ is a set-theoretic complete intersection in some positive characteristic if and only if it is a binomial set-theoretic complete intersection in the same characteristic.

From Corollary 2.3 we know that, if the dimension $n$ of $V$ is at most 2, case (ii) of Corollary 5.1 always occurs. On the contrary, for every $n \geq 3$, there are simplicial toric varieties of codimension 2 for each of the cases (i)-(iii). On the other hand, Proposition 1.10 tells us that case (ii) is fulfilled whenever the parameterization of $V$ is full. We give examples of the cases (i) and (iii) for $n=3$.

Example 5.4. Let $p$ be a prime and let $V_{T} \subset K^{5}$ be the affine toric variety attached to the set

$$
T=\{(p, 0, a),(0, p, b),(p, 0,0),(0, p, 0),(0,0, p)\},
$$

where $a$ and $b$ are positive integers not divisible by $p$. Let

$$
\tilde{T}_{1}=\{(p, 0, a)\}, \quad \tilde{T}_{2}=\{(0, p, b),(p, 0,0),(0, p, 0),(0,0, p)\} .
$$

Then

$$
\mathbb{Z}(p, 0, a)=\mathbb{Z} \tilde{T}_{1} \cap \mathbb{Z} \tilde{T}_{2} .
$$

In fact, if $\lambda$ and $\mu$ are integers such that $\lambda b+\mu p=a$, then

$$
(p, 0, a)=\lambda(0, p, b)+(p, 0,0)-\lambda(0, p, 0)+\mu(0,0, p) .
$$

On the other hand, for every positive integer $\alpha$, we have that $\alpha(p, 0, a) \in \mathbb{N} \tilde{T}_{2}$ if and only if $\alpha(p, 0, a) \in \mathbb{N}\{(p, 0,0),(0,0, p)\}$, which occurs if and only if $p$ divides $\alpha$. This shows that $T$ is the $p$-gluing of $\tilde{T}_{1}$ and $\tilde{T}_{2}$, but not the $q$-gluing of $\tilde{T}_{1}$ and $\tilde{T}_{2}$ for any prime $q \neq p$. In view of Theorem 3.1, this implies that $T$ is completely $p$-glued, but not completely $q$-glued for any prime $q \neq p$. Hence $p$ is the only positive characteristic in which $V_{T}$ is a binomial set-theoretic complete intersection; in particular, $V_{T}$ is not a complete intersection, hence, in view of Proposition 1.1, it is not a binomial settheoretic complete intersection in characteristic zero. In characteristic $p$, the variety $V_{T}$ is defined by the vanishing of the binomials

$$
F_{1}=y_{1}^{p}-x_{1}^{p} x_{3}^{a}, \quad F_{2}=y_{2}^{p}-x_{2}^{p} x_{3}^{b} .
$$

According to Remark 5.3, $V_{T}$ is not a set-theoretic complete intersection in any characteristic other than $p$. 
Example 5.5. Let $p, q$ be different primes and let $V \subset K^{5}$ be the affine toric variety attached to the set

$$
T=\{(p q, 0, a),(0, p q, b),(p q, 0,0),(0, p q, 0),(0,0, p q)\},
$$

where $a$ and $b$ are positive integers not divisible by $p$ nor $q$. Let

$$
\tilde{T}_{1}=\{(p q, 0, a)\}, \quad \tilde{T}_{2}=\{(0, p q, b),(p q, 0,0),(0, p q, 0),(0,0, p q)\} .
$$

Then

$$
\mathbb{Z}(p q, 0, a)=\mathbb{Z} \tilde{T}_{1} \cap \mathbb{Z} \tilde{T}_{2} .
$$

In fact, if $\lambda$ and $\mu$ are integers such that $\lambda b+\mu p q=a$, then

$$
(p q, 0, a)=\lambda(0, p q, b)+(p q, 0,0)-\lambda(0, p q, 0)+\mu(0,0, p q) .
$$

On the other hand, for every positive integer $\alpha$, we have that $\alpha(p q, 0, a) \in \mathbb{N} \tilde{T}_{2}$ if and only if $\alpha(p q, 0, a) \in \mathbb{N}\{(p q, 0,0),(0,0, p q)\}$, which occurs if and only if $p q$ divides $\alpha$. This shows that $T$ is the not the $p$-gluing of $\tilde{T}_{1}$ and $\tilde{T}_{2}$ for any prime $p$. In view of Theorem 3.1, this implies that $T$ is not completely $p$-glued for any prime $p$. Hence $V_{T}$ is not a binomial set-theoretic complete intersection in any characteristic.

The variety $V_{T}$ belongs to the class considered in [5, section 3] (see, in particular, Example 5). There it was shown that $V_{T}$ is in fact not a set-theoretic complete intersection in any characteristic.

Proposition 2.1 and Corollary 3.5 imply that, if $T$ is completely p-glued for some prime $p$, then $T$ is the $q$-gluing of $\tilde{T}_{1}$ and $\tilde{T}_{2}$ (or of $\tilde{\tilde{T}}_{1}$ and $\tilde{\tilde{T}}_{2}$ ) either for every prime $q$, or for no prime $q$ or only for $q=p$. The situation is different for higher codimensions. The next example, which refers to codimension 3, shows that the set

$$
T=\left\{\mathbf{a}, \mathbf{b}, \mathbf{c}, \mathbf{e}_{1}, \ldots, \mathbf{e}_{n}\right\}
$$

can admit two different partitions

$$
\begin{array}{ll}
\tilde{T}_{1}=\{\mathbf{a}\}, & \tilde{T}_{2}=\left\{\mathbf{b}, \mathbf{c}, \mathbf{e}_{1}, \ldots, \mathbf{e}_{n}\right\}, \\
\tilde{\tilde{T}}_{1}=\{\mathbf{b}\}, & \tilde{\tilde{T}}_{2}=\left\{\mathbf{a}, \mathbf{c}, \mathbf{e}_{1}, \ldots, \mathbf{e}_{n}\right\},
\end{array}
$$

with respect to which it is only $p$-glued and only $q$-glued, respectively, where $p$ and $q$ are two distinct primes.

Example 5.6. Let $V_{T} \subset K^{6}$ be the affine toric variety attached to the set

$$
T=\{(3,0,3),(0,4,2),(3,2,1),(6,0,0),(0,6,0),(0,0,6)\},
$$

and let

$$
\tilde{T}_{1}=\{(3,0,3)\}, \quad \tilde{T}_{2}=\{(0,4,2),(3,2,1),(6,0,0),(0,6,0),(0,0,6)\} .
$$


Then $\tilde{T}_{2}$ is completely $p$-glued for all primes $p$ by Proposition 2.1. Moreover,

$$
(3,0,3)=(6,0,0)+(0,6,0)+(0,0,6)-(3,2,1)-(0,4,2),
$$

whence we deduce that

$$
\mathbb{Z}(3,0,3)=\mathbb{Z} \tilde{T}_{1} \cap \mathbb{Z} \tilde{T}_{2} .
$$

For every positive integer $\alpha$, we have that $\alpha(3,0,3) \in \mathbb{N} \tilde{T}_{2}$ if and only if $\alpha(3,0,3) \in$ $\mathbb{N}\{(6,0,0),(0,0,6)\}$, which occurs if and only if 2 divides $\alpha$. This shows that $T$ is the 2 -gluing of $\tilde{T}_{1}$ and $\tilde{T}_{2}$, so that $T$ is completely 2 -glued; and it is not the $p$-gluing of $\tilde{T}_{1}$ and $\tilde{T}_{2}$ for any prime $p \neq 2$. However, if

$$
\tilde{\tilde{T}}_{1}=\{(0,4,2)\}, \quad \tilde{\tilde{T}}_{2}=\{(3,0,3),(3,2,1),(6,0,0),(0,6,0),(0,0,6)\},
$$

then $T$ is the 3-gluing of $\tilde{\tilde{T}}_{1}$ and $\tilde{\tilde{T}}_{2}$, so that $T$ is also completely 3 -glued; but it is not the $p$-gluing of $\tilde{T}_{1}$ and $\tilde{T}_{2}$ for any prime $p \neq 3$. Finally, it turns out that, for all primes $p, T$ is the $p$-gluing of

$$
\bar{T}_{1}=\{(3,2,1)\}, \quad \bar{T}_{2}=\{(3,0,3),(0,4,2),(6,0,0),(0,6,0),(0,0,6)\},
$$

where $\bar{T}_{2}$ is completely $p$-glued. Hence $T$ is completely $p$-glued for all primes $p$.

We do not know of any affine toric variety of codimension at least 3 whose attached set is completely $p$-glued for two different primes $p$, but not for all primes $p$. The above example demonstrates that proving that such varieties do not exist in codimension greater than 2 would require arguments which differ substantially from those we have applied to the case of codimension 2 .

\section{References}

[1] M. Barile, Arithmetical ranks of ideals associated to symmetric and alternating matrices, J. Algebra 176 (1995), no. 1, 59-82.

[2] _ A note on Veronese varieties, Rend. Circ. Mat. Palermo (2) 54 (2005), no. 3, 359-366.

[3] _ On toric varieties of high arithmetical rank, Yokohama Math. J. 52 (2006), no. 2, 125-130.

[4] _ On a special class of simplicial toric varieties, J. Algebra 308 (2007), no. 1, 368-382.

[5] - On simplicial toric varieties of codimension 2, Rend. Istit. Mat. Univ. Trieste (2007), to appear, available at math.AC/0705.4389.

[6] M. Barile and G. Lyubeznik, Set-theoretic complete intersections in characteristic p, Proc. Amer. Math. Soc. 133 (2005), no. 11, 3199-3209.

[7] M. Barile, M. Morales, and A. Thoma, On simplicial toric varieties which are set-theoretic complete intersections, J. Algebra 226 (2000), no. 2, 880-892.

[8] Set-theoretic complete intersections on binomials, Proc. Amer. Math. Soc. 130 (2002), no. 7, 1893-1903.

[9] H. Bresinsky, Monomial space curves in $\mathbf{A}^{3}$ as set-theoretic complete intersections, Proc. Amer. Math. Soc. 75 (1979), no. 1, 23-24. 
[10] _ Monomial Gorenstein curves in $\mathbf{A}^{4}$ as set-theoretic complete intersections, Manuscripta Math. 27 (1979), no. 4, 353-358.

[11] K. Eto, An example of set-theoretic complete intersection lattice ideal, Tokyo Math. J. 29 (2006), 319-324.

[12] K. G. Fischer, W. Morris, and J. Shapiro, Affine semigroups that are complete intersections, Proc. Amer. Math. Soc. 125 (1997), no. 11, 3137-3145.

[13] J. Heger, Denkschriften, II, Kais. Akad. Wissensch. Mathem. Naturw. Klasse 14 (1858).

[14] A. Katsabekis, Projection of cones and the arithmetical rank of toric varieties, J. Pure Appl. Algebra 199 (2005), 133-147.

[15] T. T. Moh, Set-theoretic complete intersections, Proc. Amer. Math. Soc. 94 (1985), no. 2, $217-220$.

[16] M. Morales, Équations des variétés monomiales en codimension deux, J. Algebra 175 (1995), no. 3, 1082-1095.

[17] L. Robbiano and G. Valla, On set-theoretic complete intersections in the projective space, Rend. Sem. Mat. Fis. Milano 53 (1983), 333-346.

[18] _ Some curves in $\mathbf{P}^{3}$ are set-theoretic complete intersections, Algebraic geometry (Ravello, 1982), Lecture Notes in Mathematics, vol. 997, Springer Verlag, Berlin, 1983.

[19] J. C. Rosales, On presentations of subsemigroups of $\mathbb{N}^{n}$, Semigroup Forum 55 (1997), no. 2, $152-159$.

[20] M. Şahin, Producing complete intersection monomial curves in $\mathbf{P}^{n}$ (2006), preprint, available at math. AG/0610316.

[21] A. Thoma, On the set-theoretic complete intersection problem for monomial curves in $\mathbf{A}^{n}$ and $\mathbf{P}^{n}$, J. Pure Appl. Algebra 104 (1995), 333-344.

[22] G. Valla, On determinantal ideals which are set-theoretic complete intersections, Compositio Math. 42 (1980/81), no. 1, 3-11. 